\title{
Lack of negative correlation in glucose dynamics by NEAT restriction in healthy adults
}

\author{
Hitomi Ogata ${ }^{1}$, Kazuteru Nakamura ${ }^{1}$, Maki Sato ${ }^{1}$, Kumpei Tokuyama ${ }^{1}$, Shoichiro Nagasaka ${ }^{2}$, \\ Naoyuki Ebine ${ }^{3}$, Ken Kiyono ${ }^{4}$, and Yoshiharu Yamamoto ${ }^{5}$
}

1. Graduate School of Comprehensive Human Science, University of Tsukuba, 1-1-1 Tennodai, Tsukuba-shi, Ibaraki 305-8574, Japan

2. Division of Endocrinology and Metabolism, Department of Medicine, Jichi Medical University, 3311-1 Yakushiji, Shimotsuke-shi, Tochigi 329-0498, Japan

3. Faculty of Health \& Sports Science, Doshisha University, 1-3 Tataratoya, Kyotanabe-shi, Kyoto 610-0394, Japan

4. College of Engineering General Education, Nihon University, 1 Naka-gawara, Tokusada, Tamura-machi, Koriyama-shi, Fukushima 963-8642, Japan

5. Educational Physiology Laboratory, Graduate School of Education, The University of Tokyo, 7-3-1 Hongo, Bunkyo-ku, Tokyo 113-0033, Japan

Running title: Altered glucose dynamics by NEAT restriction

Address correspondence to:

Yoshiharu Yamamoto, Ph.D.

Graduate School of Education

The University of Tokyo

7-3-1 Hongo, Bunkyo-ku

Tokyo 113-0033, Japan

Phone: +81-3-5841-3971

Fax: +81-3-5689-8069

e-mail: yamamoto@p.u-tokyo.ac.jp

Disclosure of funding: The authors declare that there is no conflict of interest associated with this manuscript. 


\begin{abstract}
Introduction: Recently, non-exercise activity thermogenesis (NEAT) has been highlighted for its ability to prevent weight gain and obesity. It has also been shown that long-range negative auto-correlation of glucose dynamics, considered to reflect long-term blood glucose controllability, breaks down in diabetic patients.
\end{abstract}

Purpose: To clarify the impact of restricted NEAT on the glycemic profile and/or control characterized by glucose auto-correlation.

Methods: Glucose dynamics of ten young healthy subjects were measured by continuous glucose monitoring (CGM) during a day with normal activity and a day with restricted NEAT. To estimate the correlation property of the glycemic fluctuation, we employed detrended fluctuation analysis (DFA), a method that analyzes the long-range temporal auto-correlation of signals.

Results: In the long-range regime (> $130 \mathrm{~min}$ ) on a normal activity day, the DFA scaling exponent was $\alpha_{2}=1.37 \pm 0.21$; this was significantly $(P=0.036)$ smaller than the reference "uncorrelated value" of $\alpha=1.5$, suggesting that glycemic fluctuation was negatively auto-correlated. In contrast, on a day with restricted NEAT in the long-range regime (> 167.5 $\min )$, the exponent was $\alpha_{2}=1.57 \pm 0.15$; this was significantly $(P=0.024)$ larger than 1.5 , implying a lack of negative correlation. 
Conclusions: The negative auto-correlation of glucose dynamics disappears with restricted NEAT compared to normal activity. This indicates that NEAT, reflective of all non-volitional muscle activity, plays an important role in long-range negative correlation and hence long-term blood glucose control in healthy young adults.

Key words: Non-exercise activity thermogenesis (NEAT), detrended fluctuation analysis (DFA), continuous glucose monitoring (CGM), glycemic fluctuation, glucose homeostasis 


\section{INTRODUCTION}

Paragraph 1: Weight gain and obesity occur when energy intake chronically exceeds energy expenditure (EE) (8). Accordingly, the balance between food intake and EE determines the body's energy stores. It is known that human EE consists of three principal components: resting metabolic rate (RMR), the thermic effect of food, and physical activity (16). RMR represents 50 to $70 \%$ of daily EE, and the absolute value seems to be "fixed" for each person and declines with age (30). Thermogenesis accounts for only 10\%. The remaining 20 to $40 \%$, therefore, is due to the most variable component: physical activity. Activity thermogenesis can be further separated into two components: exercise-related activity thermogenesis and non-exercise activity thermogenesis (NEAT). NEAT is distinct from purposeful exercise and includes the EE of daily activities such as sitting, standing, walking, and talking $(16,17,30)$.

Paragraph 2: Recently, the effectiveness of physical exercise has become a cornerstone of treatment for many chronic diseases and is often recommended in combination with dietary alterations as the initial treatment modality in newly diagnosed individuals with metabolic syndrome and type 2 diabetes $(4,40)$. Activities of moderate intensity, such as brisk walking, have been associated with reduced risk of coronary heart disease (10), stroke (9), and type 2 diabetes (12). This is primarily due to beneficial effects on body weight, blood pressure, serum 
cholesterol, and glucose tolerance. Compared to lean people, obese individuals have dramatically reduced spontaneous physical activity and thus, have reduced NEAT $(16,30)$.

Paragraph 3: In the past decade, various technologies allowing for ambulatory continuous glucose monitoring (CGM) have become available, in addition to self-monitoring of blood glucose (SMBG). CGM can provide "real life" information on glucose profiles, including the effects of exercise and NEAT, measured individually in daily life (11). Moreover, the effectiveness of using CGM when counseling diabetic patients on physical activity has been reported in several papers $(18,19,29)$. Although CGM is now widely used in clinical settings, the wealth of data taken from CGM has yet to be fully utilized (22).

Paragraph 4: It is well known that normal glucose homeostasis is achieved, through a negative feedback regulation, by a tightly controlled balance between glucose flux and glucose reflux (38). In addition, there is growing evidence that physiological signals often display a complex (power-law type) negative auto-correlation $(25,28)$ rather than an exponential one, which would be expected for standard linear negative feedback systems. In fact, we recently showed that glucose dynamics measured in ambulatory settings using CGM are non-stationary and exhibit substantial complexity (26), precluding the use of standard time series analytical techniques. We evaluated glucose dynamics in humans using detrended fluctuation analysis (DFA) to detect long-range auto-correlations in noisy and non-stationary time series (28) and demonstrated a 
breakdown of the long-range negative correlation of CGM-based glucose dynamics in diabetic patients (26). Thus it can be argued that the normal long-term glucose homeostasis is a nontrivial and far more complex system than that which is explained by the linear feedback regulation. Moreover, the increase in the long-range DFA scaling exponent, indicative of the lack of long-range negative auto-correlation in glucose dynamics, reflects abnormalities in average glycemic control clinically evaluated by HbAlc and glycated albumin (27).

Paragraph 5: Despite the possibility that obese individuals have both lower NEAT $(16,30)$ and worse glucose controllability (6), however, there is no reported influence of physical activity, including NEAT, on glycemic fluctuation. We hypothesized that glucose homeostasis is affected not only by physical activity, but also by restricted NEAT. Therefore, the purpose of this study was to clarify the impact of restricted NEAT on glycemic fluctuation as measured by DFA in healthy young subjects. Numerous indices for the quality of glycemic control and glycemic variability have been proposed previously (32). We predicted that the effect of NEAT might not only be observed in the DFA indices, but also in some of the ordinary indices of glycemic control. Accordingly, we also examined ordinary methods of glycemic instability to evaluate glycemic control in conjunction with DFA. 


\section{METHODS}

Paragraph 6: Subjects. Ten healthy young subjects (5 males and 5 females, age: $24.5 \pm 3.0$ yrs., body mass index (BMI): $20.6 \pm 2.0 \mathrm{~kg} / \mathrm{m}^{2}$, fasting glucose level measured by CGM: $78.1 \pm 9.4$ $\mathrm{mg} / \mathrm{dl}$ ) participated in this study. We excluded smokers, diabetes mellitus patients, patients with impaired glucose tolerance (IGT) and physical disability. All the subjects were in good health, had no known medical disorders and took no medication. The study was approved by the local ethics committee at the University of Tsukuba, and all the subjects gave their informed consent to participate.

Paragraph 7: Measurements. Glucose dynamics were measured by a retrospective type CGM (CGMS Gold, Medtronic MiniMed, Northridge, CA, USA) comprised of a Holter-style sensor system; this has been described in detail previously (21). CGM is designed to monitor subcutaneous interstitial fluid glucose concentrations within a range of 40 to $400 \mathrm{mg} / \mathrm{dL}$ and the subjects are required to SMBG at least 4 times a day for calibration purposes. The current is carried by a cable to a pager-size monitor that analyzes the data every $10 \mathrm{~s}$, reports average values every $5 \mathrm{~min}$, and gives a total of 288 readings per day.

Paragraph 8: Physical activity, including NEAT, was objectively measured the ActiGraph uniaxial accelerometer activity monitor (Ambulatory Monitoring Inc. NY, USA). All subjects 
wore the wrist-watch accelerometer with zero-crossing mode (2). Gross motor activity of the accelerometer was estimated at one-minute intervals.

Paragraph 9: Protocols. Subjects participated in two trials in a randomized repeated-measures design: 1) The subjects pursued their normal activities day with their physical activity factor of 1.5 (moderate), and refrained from exhaustive physical activity; 2) The subjects limited their non-volitional muscle activities (i.e., restricted NEAT). Each trial was conducted in a 1-month cycle for female subjects, and we confirmed that they participated in the study in the same menstrual phase, i.e., luteal or follicle phase. Male subjexts were tested in 2-week cycle. The subjects followed a consistent daily schedule. They woke up (06:00), had meals (07:00, 12:00 and 19:00), and went to sleep (23:00) at the same time each day during the measurement period. The size of the standardized meals was individually adjusted based on estimated energy requirement for Japanese persons (23) assuming a physical activity factor of 1.5. Average energy content of the standardized meals was $2167 \pm 59 \mathrm{kcal} /$ day $(722 \pm 22 \mathrm{kcal} / \mathrm{meal})$. Expressed as a percentage of total energy, the meals contained $16.8 \%$ protein, $19.9 \%$ fat, and $63.3 \%$ carbohydrate. Subjects ate nothing between meals, abstained from alcohol and caffeine intake for 24 hours before the study and abstained from exhaustive exercise for 48 hours prior to the study. When subjects were undergoing NEAT restriction, they spent the trial in a room-sized chamber that had an internal volume of $14.49 \mathrm{~m}^{3}(2.00 \times 3.45 \times 2.10 \mathrm{~m})$, which was furnished with an 
adjustable hospital bed, a desk, a chair, and a toilet, and subjects restricted all of their activities except using the toilet, eating meals, and sleeping.

Paragraph 10: DFA. To estimate the correlation property of glucose fluctuation, we used an extended random walk analysis (28) called DFA. DFA has been described in detail previously $(26,27)$. The advantage of DFA is that it can more accurately quantify the temporal correlation property of original signals, even if those signals are masked by nonstationarity compared with traditional methods such as standard auto-correlation and/or power spectrum analysis (37). First, we integrated the glucose time series data. Next, we prepared equally sized sliding (one point at a time) windows of length n. For each window, the third-order polynomial trend, representing nonstationarity in that window, was fit to the data. The detrended fluctuations $F(n)$ were then calculated as the root-mean-square deviation from the trend in each window, which was summed for all of the windows of the entire time series analyzed. This procedure was repeated for different window sizes (scales). We used DFA to calculate the short-range scaling exponent $\left(\alpha_{1}\right)$, the long-range scaling exponent $\left(\alpha_{2}\right)$, and the mean glucose fluctuation (the averaged $\log _{10} \mathrm{Fm}$ ).

Paragraph 11: In this study, the range of scales applied was from $\log _{10} n \sim 0.90$ to $\log _{10} n \sim 1.85$, which corresponds to a range of 40-350 min. Uncorrelated white noise yields $\alpha=0.5$ and Brownian motion (i.e., integrated white noise with uncorrelated increments or changes) yields $\alpha$ $=1.5$. Therefore, the long-range negatively correlated fluctuation in glucose level is represented 
by $\alpha<1.5$ for the measured glucose time series, while the positively correlated fluctuation is represented by $\alpha>1.5$.

Paragraph 12: Glycemic instability. Several approaches have been used to assess glucose homeostasis to express the degree of glucose regulation and/or glycemic instability in diabetic patients. M-value: This is obtained by a logarithmic transformation of the deviation of glycemia from an arbitrarily selected value $(90 \mathrm{mg} / \mathrm{dl}$ in this study) plus an amplitude correction factor (34). Mean Amplitude of Glycemic Excursions (MAGE): This is obtained by averaging glycemic excursions exceeding 1 standard deviation (SD) (35). Mean Indices of Meal Excursions (MIME): This describes postprandial glycemic events with two indices composed of time from the start of the meal to the peak postprandial glycemia $(\Delta \mathrm{T})$ and the rise in glucose from pre-prandial to peak postprandial levels $(\Delta \mathrm{G})$ (36). Continuous Overall Net Glycemic Action (CONGAn): This is defined as the SD of all the differences, after the first $n$ hours, between the current observation and the observation $n$ hours (1,2, and 4) before (22).

Paragraph 13: Statistical analyses. All data are expressed as the mean \pm SD. Differences in the short- and long-range scaling exponents within and between normal activity and restricted NEAT days, those from the uncorrelated "reference" value of $\alpha=1.5$, and differences in the $F m$, physical activity, and ordinary glycemic indices between the two trials were evaluated using Student's $t$-tests. The DFA plots (i.e., plots of $\log _{10} n$ vs. $\left.\log _{10} F(n)\right)$ for subjects exhibit the 
"crossover" phenomenon; there is a difference in the glucose correlation properties between $\alpha_{1}$ and $\alpha_{2} . \alpha_{1}, \alpha_{2}$, and the crossover point were determined for each subject and each trial by the best

two-line fit based on the $\chi^{2}$-test and on the Akaike information criterion (AIC) (1). The level of statistical significance was set at $P<0.05$.

\section{RESULTS}

Paragraph 14: Representative example. Figure 1 illustrates representative records of glucose dynamics and the resultant DFA plots (Figure 1A) and activity levels (Figure 1B) of a healthy young subject (BMI, $20.8 \mathrm{~kg} / \mathrm{m}^{2}$ ). The glucose and physical activity dynamics are shown for a normal activity day $(91.2 \pm 18.6 \mathrm{mg} / \mathrm{dl}, 138.5$ counts $/ \mathrm{min}$; black lines $)$ and a restricted NEAT day $(93.4 \pm 18.3 \mathrm{mg} / \mathrm{dl}, 86.9$ counts/min; gray lines) during a typical daily schedule with meals and sleep. Physical activities are shown after a 5-min moving average because raw data (1-min time intervals) contained too many high frequency components, and they indicate that the subject maintained the same time schedules during the two trials but has lower activity on the restricted NEAT day. The DFA plots for the normal activity day and the restricted NEAT day exhibit a crossover phenomenon $\left(\alpha_{1}=2.53\right.$ and $\alpha_{2}=1.36, \alpha_{1}=2.33$ and $\alpha_{2}=1.51$, respectively, inset of Figure 1A), indicative of the lack or weakening of long-range negative correlation on the restricted NEAT day. 
Paragraph 15: Comparison between trials. Physical activity level and ordinary indices for glycemic variability between the two trials are shown in Table 1. In comparison with a normal activity day, the mean glucose levels and the SD of glucose levels were not significantly different on a restricted NEAT day. Meanwhile, the difference in overall physical activity including NEAT of more than $0.01 \mathrm{G} / \mathrm{Rad} / \mathrm{sec}$ reached statistical significance $(P=0.039)$; these counts for the normal activity day with moderate activity intensity are similar to those reported in another study with healthy population $(15)$. Moreover, $\Delta \mathrm{G}$ of the MIME $(P=0.039)$ and CONGA1 $(P=0.038)$ were significantly different between these trials.

Paragraph 16: Glycemic fluctuation. The DFA plots for healthy young subjects for a normal activity day (Fig. 2A), for a restricted NEAT day (Fig. 2B), and for the trial averages $F(n)$ (Fig. 2C) are shown in Figure 2. The two-line regression placed a crossover point for the grouped data at approximately $130 \mathrm{~min}$, and the individual crossover points fell in a range from 110 to 215 min on a normal activity day. On the other hand, the individual crossover points for a restricted NEAT day fell in a range from 62.5 to $235 \mathrm{~min}$, while the crossover point for the trial data occurred at approximately $167.5 \mathrm{~min}$. Compared with a normal activity day, $\mathrm{Fm}$ were not significantly different $(0.638 \pm 0.16$ vs. $0.648 \pm 0.17, P>0.05)$ on a restricted NEAT day. In the short-range regime $(<130 \mathrm{~min})$, the DFA scaling exponent for a normal activity day was $\alpha_{1}=$ $2.00 \pm 0.39(P=0.002$ for the difference compared to the "uncorrelated reference value" of $\alpha=$ 
1.5). For a restricted NEAT day in the short-range regime ( $<167.5 \mathrm{~min})$, the scaling exponent was $\alpha_{1}=2.14 \pm 0.40(P=0.001$ for the difference compared to $\alpha=1.5)$. The scaling exponents of the two trials were not significantly different (Table 1). These results imply that the net effects of glucose flux/reflux persist within these shorter timescales. In addition to these short timescales, the scaling exponent was $\alpha_{2}=1.57 \pm 0.15(P=0.024$ for the difference compared to $\alpha=1.5)$ on a restricted NEAT day in the long-range regime (> $167.5 \mathrm{~min})$. This suggests that, even in the long-range regime, weakened negative feedback on a restricted NEAT day cause a persistence of the net effect of the flux and reflux for many hours in glycemic dynamics. On the other hand, in the long-range regime (>130 min) on a normal activity day, the scaling exponent was $\alpha_{2}=1.37$ \pm 0.21 , suggesting that glycemic fluctuation was negatively auto-correlated $(P=0.036$ for the difference compared to $\alpha=1.5$ ) and the long-range scaling exponents were significantly different between the two trials (Table 1). This means that the glucose level is tightly regulated by a negative feedback mechanism in these longer timescales on a normal activity day, giving rise to long-term stability.

\section{DISCUSSION}

Paragraph 17: It has been established that a single period of physical exercise can improve glycemic profiles $(4,40)$ and many experts in the field believe that CGM will increase the 
likelihood that physically active people with diabetes can actually improve overall metabolic control (31). It is reported that obesity is associated with low $\operatorname{NEAT}(16,30)$; however, there is no reported influence of physical activity, including NEAT, on glycemic fluctuation. The present study, therefore, investigated whether restriction of NEAT affects glycemic fluctuation in healthy young subjects using DFA (28), a multi-scale temporal correlation analysis of the glycemic profile, together with ordinary measures to qualify glycemic control. In the long-range regime, the scaling exponent $\alpha_{2}$ derived from the DFA for a normal activity day indicated the increased probability of switching the direction of glycemic fluctuation because a scaling exponent value lower than 1.5 implies long-range negative auto-correlation, or anti-persistency. On the other hand, on a restricted NEAT day, both in the short- and long-range regimes, $\alpha$ was higher than 1.5, implying that the net effects of the flux and reflux persist for many hours, giving rise to a weaker negative feedback mechanism. Thus, the present findings show that all non-volitional muscle activities, reflected by NEAT, might play an important role in glucose homeostasis in healthy young subjects by ensuring long-range negative auto-correlation in glucose dynamics.

Paragraph 18: Glycemic fluctuation. Blood glucose level reflects the balance between glycogenolysis (including glycolysis) and gluconeogenesis in the skeletal muscles and the liver. Under healthy conditions, exercise initiates simultaneous elevations in hepatic glucose production and glucose utilization. This relatively simple and effective relationship between the 
hepatic and muscular effects is maintained by a complex interplay of circulating and locally released neuroendocrine controllers. Physical exercise can improve insulin sensitivity (diminishes resistance) and achieve (maintain) near-normal glucose levels and optimal lipid levels in type 2 diabetes $(4,40)$. Even acute bouts of endurance $(7,18)$ or resistance exercise $(13)$ have been shown to improve insulin sensitivity and/or glucose tolerance. These effects have been reported to persist for a period ranging from 2 hours (24), 4-6 hours (39), 12-16 hours (7), 24 hours $(13,18,19,29)$, and up to 48 hours after cessation of exercise (24). Moreover, in the present study, light and non-volitional muscle activities (i.e., NEAT) were shown to affect glycemic fluctuation, although we did not perform detailed analyses on insulin sensitivity or glucose tolerance.

Paragraph 19: Relationship with NEAT. NEAT may be related to BMI in the non-obese stage. Participants with higher NEAT have a lower BMI than their peers, $t$ i.e., an inverse correlation of the walking distance with the BMI (5). The present results indicate that glucose control, shown by glycemic fluctuation, is also under the influences of the NEAT components. Marra et al. studied the regulatory roles of NEAT including its protective role to prevent excess body fat accumulation in women of different weight (20). They measured body composition, RMR, fidgeting as a component of NEAT, and respiratory quotient, an index of preferential substrate oxidation. It was shown that NEAT, and particularly fidgeting, facilitates maintenance of a lean 
body type and could be protective against excess body fat deposition (20). Moreover, fidgeting in the lean people was associated with a preferential fat oxidation, while it was inversely related to fat oxidation in obese people despite large fat stores.

Paragraph 20: The mechanisms regulating NEAT are not completely understood, but the sympathetic nervous system seems to play an important role (3). Recent evidence indicates that spontaneous physical activity and the resulting thermogenesis (NEAT) may be regulated by the brain $(14,33)$. Thus, orexin neurons, widespread projections and connectivity to other brain areas responsible for energy homeostasis, which are active in both food intake and physical activity (whether it is spontaneous or volitional), are well suited to perform an integrative function (14). It has been reported that overfeeding and fat gain results in reduced NEAT (17). The present study showed that glucose homeostasis becomes weaker with restricted NEAT in healthy young subjects. It is expected that this effect might be even larger in middle-aged subjects and/or obese subjects.

Paragraph 21: Relationship with ordinary indices. Several approaches have been employed when analyzing blood glucose data to assess the degree of glycemic instability and to facilitate monitoring and diagnosis of glucose regulatory system dysfunction. Among these indices, there were significant differences between the two trials in only two indices, $\Delta \mathrm{G}$ of the MIME and CONGA1. These results suggest that glucose dynamics become large at short-range regime and 
postprandial glycemia is increased when restricting NEAT. Although the mean glucose and the SD for 24 hours were not different between two trials, the difference in glucose dynamics was reflected in the DFA exponent. We concluded that the effect of NEAT on glycemic fluctuation might not only be observed in the DFA indices, but also in some of the ordinary indices for glycemic control. It is further speculated that mechanisms responsible for lower postprandial glycemia and/or glucose excursion within 1 hour may be related to the long-range negative auto-correlation shown by the DFA exponent that is reported to be lost in diabetic patients (26, 27).

Paragraph 22: This study has some limitations. First, we did not conduct blood sampling, physiological data by blood sampling may explain the mechanism of glucose metabolism. Second, the sample size was small. When we calculate a sample size for a power of 0.8 with $\alpha=$ 0.05 using the data of the present study, the sample size needed is at least 16 for each comparison, in order to demonstrate a significant difference in physical activity, $\Delta \mathrm{G}$ of the MIME, and CONGA1 using paired $t$-test. Third, the subjects were young and healthy. Therefore, our results cannot be extrapolated to other populations, such as older, obese, or type 2 diabetic populations. Lastly, we only measured acute effects of restricted NEAT instead of measuring chronic effects. In the present study, however, we could indicate the importance of NEAT for young healthy 
subjects, and the importance in patients with IGT and/or diabetes mellitus should be carefully

evaluated in the future.

Paragraph 23: In conclusion, a weaker long-range negative auto-correlation in glucose dynamics was associated with restricted NEAT in healthy young adults. This might suggest a possible role of all non-volitional muscle activities, reflected by NEAT, in the control of glucose levels. It is speculated that the same role played by NEAT might be important for normal glucose fluctuation or glucose homeostasis in patients with metabolic syndrome and/or that of IGT.

\section{ACKNOWLEDGMENTS}

Paragraph 24: The authors disclose that no funding was received for this work. All authors report no conflicts of interest. The results of the present study do not constitute an endorsement by the American College of Sports Medicine. The authors thank all the subjects who volunteered to participate in this study for their enthusiastic support.

\section{CONFLICTS OF INTEREST}

Paragraph 25: None.

\section{REFERENCES}

1. Akaike H. Information theory and an extension of the maximum likelihood principle. Budapest, Akademiai Kiado; 1973, p 261-81. 
2. Ancoli-Israel S, Cole R, Alessi C, Chambers M, Moorcroft W, Pollak CP. The role of actigraphy in the study of sleep and circadian rhythms. Sleep. 2003;26(3):342-92.

3. Astrup A, Simonsen L, Bülow J, Madsen J, Christensen NJ. Epinephrine mediates facultative carbohydrate-induced thermogenesis in human skeletal muscle. Am J Physiol. 1989;257(3 Pt 1):E340-5.

4. Colberg SR, Albright AL, Blissmer BJ, Braun B, Chasan-Taber L, Fernhall B, Regensteiner JG, Rubin RR, Sigal RJ; American College of Sports Medicine; American Diabetes Association. Exercise and type 2 diabetes: American College of Sports Medicine and the American Diabetes Association: joint position statement. Exercise and type 2 diabetes. Med Sci Sports Exerc. 2010;42(12):2282-303.

5. Conzett-Baumann K, Jaggi GP, Hüsler A, Hüsler J, Beer JH. The daily walking distance of young doctors and their body mass index. Eur J Intern Med. 2009;20(6):622-4.

6. Cunningham SA, Riosmena F, Wang J, Boyle JP, Rolka DB, Geiss LS. Decreases in diabetes-free life expectancy in the U.S. and the role of obesity. Diabetes Care. 2011;34(10):2225-30.

7. Devlin JT, Hirshman M, Horton ED, Horton ES. Enhanced peripheral and splanchnic insulin sensitivity in NIDDM men after single bout of exercise. Diabetes. 1987;36(4):434-9. 
8. Hill JO, Wyatt HR, Reed GW, Peters JC. Obesity and the environment: where do we go from here? Science. 2003;299(5608):853-5.

9. Hu FB, Stampfer MJ, Colditz GA, Ascherio A, Rexrode KM, Willett WC, Manson JE. Physical activity and risk of stroke in women. JAMA. 2000;283(22):2961-7.

10. Hu G, Jousilahti P, Borodulin K, Barengo NC, Lakka TA, Nissinen A, Tuomilehto J. Occupational, commuting and leisure-time physical activity in relation to coronary heart disease among middle-aged Finnish men and women. Atherosclerosis. 2007;194(2):490-7.

11. Klonoff DC. Continuous glucose monitoring: roadmap for $21^{\text {st }}$ century diabetes therapy. Diabetes Care. 2005;28(5):1231-9.

12. Knowler WC, Barrett-Connor E, Fowler SE, Hamman RF, Lachin JM, Walker EA, Nathan DM; Diabetes Prevention Program Research Group. Reduction in the incidence of type 2 diabetes with lifestyle intervention or metformin. N Engl J Med. 2002;346(6):393-403.

13. Koopman R, Manders RJ, Zorenc AH, Hul GB, Kuipers H, Keizer HA, van Loon LJ. A single session of resistance exercise enhances insulin sensitivity for at least $24 \mathrm{~h}$ in healthy men. Eur J Appl Physiol. 2005;94(1-2):180-7.

14. Kotz CM. Integration of feeding and spontaneous physical activity: role for orexin. Physiol Behav. 2006;88(3):294-301. 
15. Leidy NK, Abbott RD, Fedenko KM. Sensitivity and reproducibility of the dual-mode actigraph under controlled levels of activity intensity. Nurs Res. 1997;46(1):5-11.

16. Levine JA, Eberhardt NL, Jensen MD. Role of nonexercise activity thermogenesis in resistance to fat gain in humans. Science. 1999;283(5399):212-4.

17. Levine JA. Nonexercise activity thermogenesis (NEAT): environment and biology. Am $J$ Physiol. 2004;286(5):E675-85.

18. MacDonald AL, Philp A, Harrison M, Bone AJ, Watt PW. Monitoring exercise-induced changes in glycemic control in type 2 diabetes. Med Sci Sports Exerc. 2006;38(2):201-7.

19. Manders RJ, Van Dijk JW, van Loon LJ. Low-intensity exercise reduces the prevalence of hyperglycemia in type 2 diabetes. Med Sci Sports Exerc. 2010;42(2):219-25.

20. Marra M, Pasanisi F, Montagnese C, De Filippo E, De Caprio C, de Magistris L, Contaldo F. BMR variability in women of different weight. Clin Nutr. 2007;26(5):567-72.

21. Mastrototaro J. The MiniMed Continuous Glucose Monitoring System (CGMS). J Pediatr Endocrinol Metab. 1999;12(Suppl 3):751-8.

22. McDonnell CM, Donath SM, Vidmar SI, Werther GA, Cameron FJ. A novel approach to continuous glucose analysis utilizing glycemic variation. Diabetes Technol Ther. 2005;7(2):253-63. 
23. Ministry of Health, Labour and Welfare, Japan, Dietary Reference Intakes for Japanese, 2010, Tokyo, Japan: Daiichi Shuppan; 2005.

24. Mikines KJ, Sonne B, Farrell PA, Tronier B, Galbo H. Effect of physical exercise on sensitivity and responsiveness to insulin in humans. Am J Physiol. 1988;254(3 Pt 1):E248-59.

25. Nunes Amaral LA, Ivanov PC, Aoyagi N, Hidaka I, Tomono S, Goldberger AL, Stanley HE, Yamamoto Y. Behavioral-independent features of complex heartbeat dynamics. Phys Rev Lett. 2001;86(26 Pt 1):6026-9.

26. Ogata H, Tokuyama K, Nagasaka S, et al. Long-range negative correlation of glucose dynamics in humans and its breakdown in diabetes mellitus. Am $J$ Physiol. 2006;291(6):R1638-43.

27. Ogata $\mathrm{H}$, Tokuyama $\mathrm{K}$, Nagasaka $\mathrm{S}$, et al. The lack of long-range negative correlations in glucose dynamics is associated with worse glucose control in diabetes mellitus patients. Metabolism, in press.

28. Peng CK, Havlin S, Stanley HE, Goldberger AL. Quantification of scaling exponents and crossover phenomena in nonstationary heartbeat time series. Chaos. 1995;5(1):82-7.

29. Praet SF, Manders RJ, Lieverse AG, Kuipers H, Stehouwer CD, Keizer HA, van Loon LJ. Influence of acute exercise on hyperglycemia in insulin-treated type 2 diabetes. Med Sci Sports Exerc. 2006;38(12):2037-44. 
30. Ravussin E. Physiology. A NEAT way to control weight? Science. 2005;307(5709):530-1.

31. Riddell M, Perkins BA. Exercise and glucose metabolism in persons with diabetes mellitus: perspectives on the role for continuous glucose monitoring. J Diabetes Sci Technol. 2009;3(4):914-23.

32. Rodbard D. Interpretation of continuous glucose monitoring data: glycemic variability and quality of glycemic control. Diabetes Technol Ther. 2009;11(Suppl 1):S55-67.

33. Sakurai T. Orexin: a link between energy homeostasis and adaptive behaviour. Curr Opin Clin Nutr Metab Care. 2003;6(4):353-60.

34. Schlichtkrull J, Munck O, Jersild M. The M-value, an index of blood-sugar control in diabetics. Acta Med Scand. 1965;177:95-102.

35. Service FJ, Molnar GD, Rosevear JW, Ackerman E, Gatewood LC, Taylor WF. Mean amplitude of glycemic excursions, a measure of diabetic instability. Diabetes. 1970;19(9):644-55.

36. Service FJ, Nelson RL. Characteristics of glycemic stability. Diabetes Care. 1980;3(1):58-62.

37. Taqqu MS, Teverovsky V, Willinger W. Estimators for long-range dependence: an empirical study. Fractals. 1995;3(4):785-98.

38. Van Cauter E, Polonsky KS, Scheen AJ. Roles of circadian rhythmicity and sleep in human glucose regulation. Endocr Rev. 1997;18(5):716-38. 
39. Wojtaszewski JF, Hansen BF, Gade, Kiens B, Markuns JF, Goodyear LJ, Richter EA. Insulin signaling and insulin sensitivity after exercise in human skeletal muscle. Diabetes. 2000;49(3):325-31.

40. Zinman B, Ruderman N, Campaigne BN, Devlin JT, Schneider SH; American Diabetes Association. Physical activity/exercise and diabetes. Diabetes Care. 2004;27(Suppl 1):S58-62.

\section{Figure captions}

Figure 1: Representative records of glucose dynamics and the resultant DFA plots (A) and physical activity (B) of a healthy young subject (BMI, $20.8 \mathrm{~kg} / \mathrm{m}^{2}$ ), as measured by CGM and ActiGraph. The black lines show data for a normal activity day and the gray lines show data for a restricted NEAT day. These data are representative of the responses to a typical daily schedule with meals and sleep.

Figure 2: DFA plots for ten healthy young subjects for a normal activity day (A), for a restricted NEAT day (B), and the trial mean fluctuation functions $F(n)(\mathrm{C})$; plots of trial means with error bars representing the SD. 
Table 1. ActiGraph and CGM measurement during normal activity day and restricted NEAT day.

\begin{tabular}{lccc}
\hline$n=10$ & Normal activity day & Restricted NEAT day & $P$-value \\
\hline \hline Physical activity (counts/min) & $126.8 \pm 19.4$ & $100.2 \pm 22.6$ & $0.039^{*}$ \\
\hline Ordinary indices & & & \\
Mean 24-h glucose (mg/dl) & $83.0 \pm 6.4$ & $80.9 \pm 6.9$ & 0.375 \\
24-h SD (mg/dl) & $11.0 \pm 4.7$ & $13.0 \pm 5.3$ & 0.211 \\
M-value & $0.94 \pm 0.75$ & $1.04 \pm 0.68$ & 0.786 \\
MAGE & $25.6 \pm 15.1$ & $33.8 \pm 15.4$ & 0.158 \\
MIME & & & \\
$\quad \Delta$ T (min) & $47.8 \pm 13.5$ & $41.8 \pm 7.5$ & 0.083 \\
$\quad \Delta$ G (mg/dl) & $29.2 \pm 15.4$ & $37.6 \pm 15.7$ & $0.039^{*}$ \\
CONGA $n$ & & & \\
$\quad$ CONGA1 & $0.677 \pm 0.31$ & $0.849 \pm 0.35$ & $0.038^{*}$ \\
$\quad$ CONGA2 & $0.932 \pm 0.47$ & $1.187 \pm 0.49$ & 0.060 \\
$\quad$ CONGA4 & $1.015 \pm 0.47$ & $1.206 \pm 0.48$ & 0.174 \\
\hline DFA & & & \\
$\alpha_{1}$ (short-range scaling exponent) & $2.00 \pm 0.39$ & $2.14 \pm 0.40$ & 0.302 \\
$\alpha_{2}$ (long-range scaling exponent) & $1.37 \pm 0.21$ & $1.57 \pm 0.15$ & $0.000^{*}$ \\
Fm & $0.638 \pm 0.16$ & $0.648 \pm 0.17$ & 0.793 \\
\hline Da
\end{tabular}

Data are presented as the mean \pm SD. CONGA $n$, continuous overall net glycemic action: the SD of all the differences, after the first $n$ hours, between the current observation and the observation $n$ hours (1, 2, and 4) before; DFA, detrended fluctuation analysis; M-value: the deviation of glycemia from an arbitrarily selected value $(90 \mathrm{mg} / \mathrm{dl}$ in this study) plus an amplitude correction factor; MAGE, mean amplitude of glycemic excursions: averaging glycemic excursions exceeding 1 standard deviation; MIME, mean indices of meal excursions (MIME): $\Delta \mathrm{T}$ : time from the start of the meal to the peak postprandial glycemia, $\Delta \mathrm{G}$ : the rise in glucose from pre-prandial to peak postprandial levels.

${ }^{*}$ Significant difference, $P<0.05$, Student's $t$-test. 

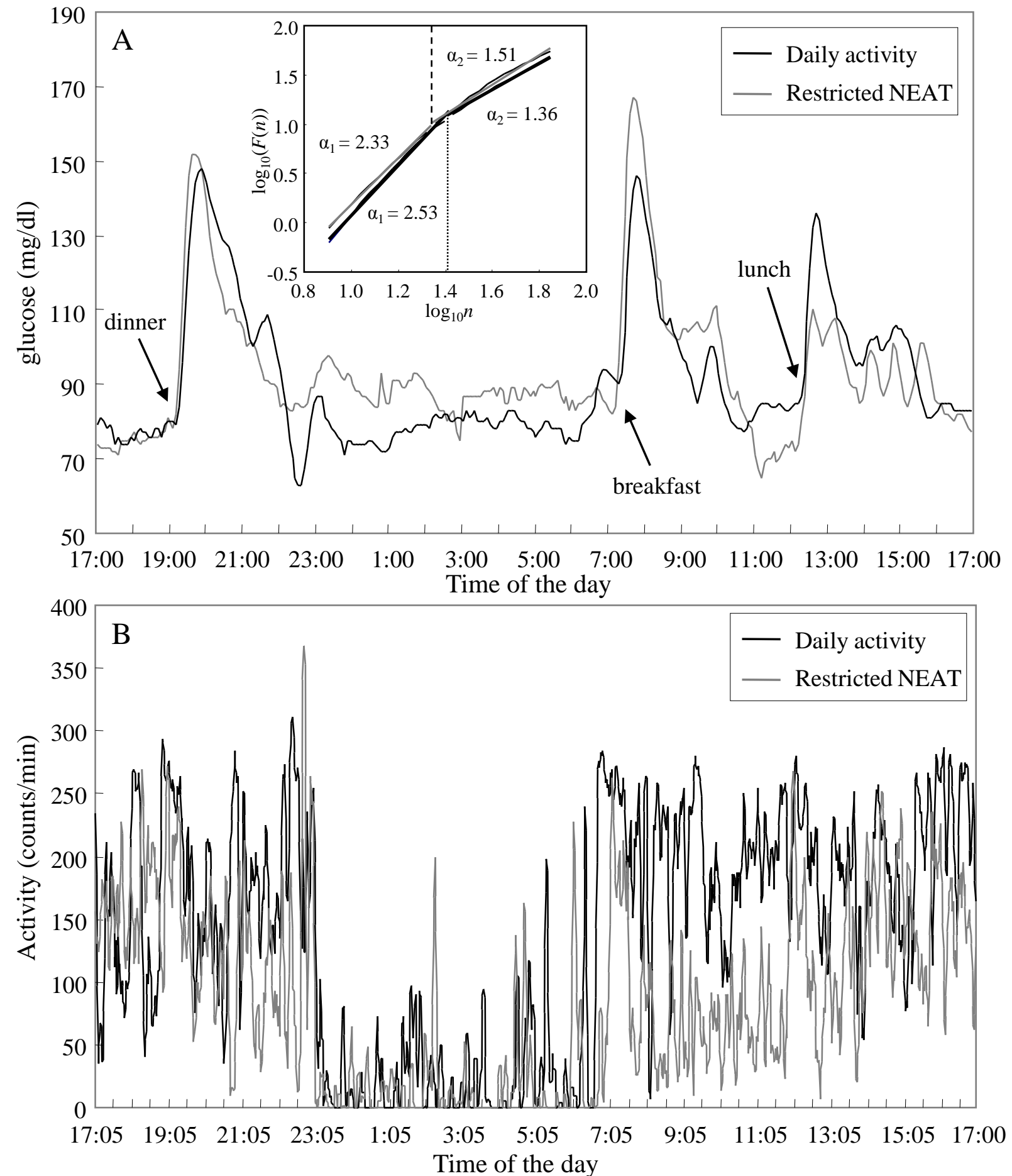

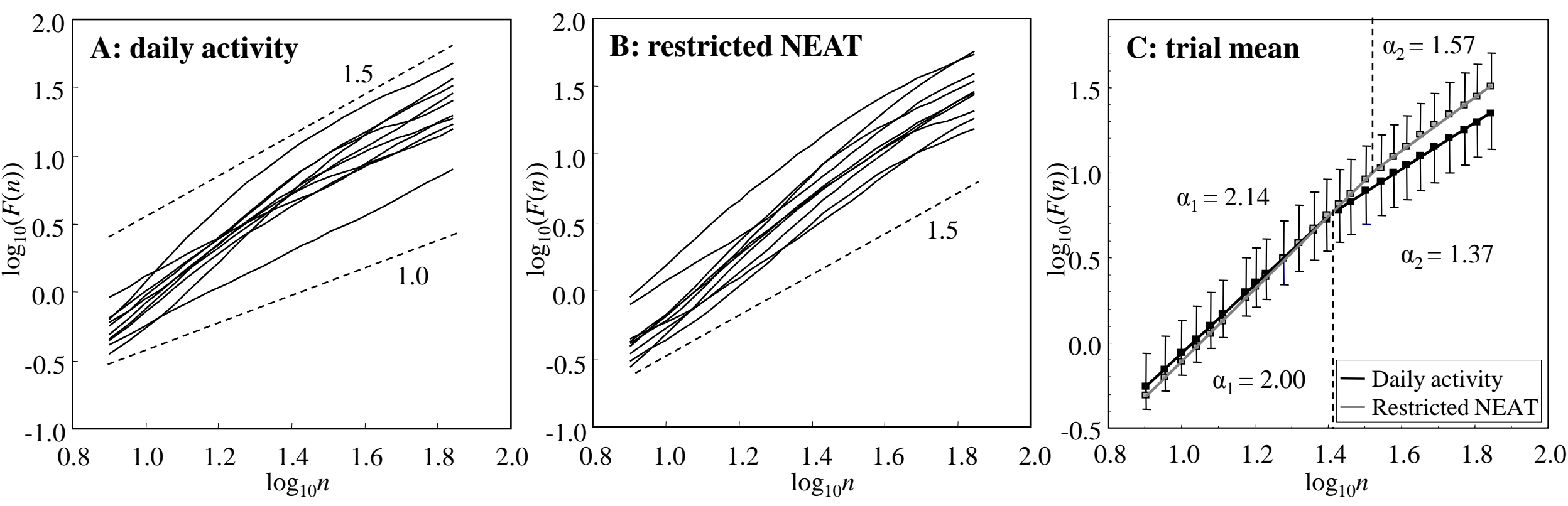